\title{
Surface and Edge Information for Spatial Integration: A Saccadic-selection Search Task
}

\author{
Iain D. Gilchrist \\ Department of Experimental Psychology, University of Bristol, UK \\ John M. Findlay and Charles A. Heywood \\ Centre for Vision and Visual Cognition, Department of Psychology, Durham \\ University, $U K$
}

\begin{abstract}
Three experiments are described that investigate visual integration across space using a saccade selection paradigm. Subjects saccaded to a vertical target in the presence of a number of horizontal distractor items. Both horizontal and vertical items were composed of two identical elements, so, in order to locate the target, subjects had to integrate the pairs of elements together. We measured saccade accuracy, the proportion of saccades directed to the vertical target, together with saccade latency following display appearance. In Experiment 1 we found that saccade accuracy was improved by the items having either common surface properties or collinear edges. These effects were not a result of increased display heterogeneity (Experiment 2), or a result of the introduction of a strong internal contour between the items (Experiment 3 ). These results show that for saccadic selection both surface and edge properties of items are processed. This in turn suggests that early visual processing encodes and exploits both types of information.
\end{abstract}

\section{INTRODUCTION}

An ongoing debate in vision research is whether early visual processes encode and utilize primarily edge information (e.g. Marr \& Hildreth, 1980), or surface information (e.g. He \& Nakayama, 1992) or some composite that retains both edge and surface information for active processing (e.g. Grossberg \& Mingolla, 1985; Watt \& Morgan, 1985).

Dr. I.D. Gilchrist, Deptartment of Experimental Psychology, University of Bristol, 8 Woodland Road, Bristol, BS8 1TN, UK; or by email: i.d.gilchrist@ bristol.ac.uk

This work was supported by a grant from the Medical Research Council (UK). We are grateful to Dr R. Kentridge for developing both the display and the eye-movement software and to Dr F. Newell for comments on an earlier draft of this paper. 
One method of resolving this issue is to study the dependence of a particular early visual process on surface and edge information. For example, in this study we investigated whether spatial integration depends on surface and edge information. If it can be shown that spatial integration is sensitive to both edge and surface properties of the items (to be integrated) then this suggests that both types of information are encoded and exploited by early visual processes. This study follows an earlier piece of work (Gilchrist, Humphreys, Riddoch, \& Neumann, 1997) in which it was shown that both edge and surface information can be used in a reaction time based visual search task. The current study investigates whether the same spatial integration processes are also used for target localization by saccades.

\section{Spatial Integration}

Perceptual integration encompasses a range of interesting phenomena. In general, these involve the combination of information to form a new unitary whole. One example is Triesman's Feature Integration Theory (e.g. Triesman, 1988), which is concerned with the processes by which the features associated with an object (e.g. its colour and form) become combined. Here we are interested in a specific, but different, kind of visual integration- spatial integration. These are the processes by which elements that are spatially separated become integrated. This is also often called perceptual grouping. In many cases, grouping can be seen as providing a perceptual link between items that are spatially separated. Gilchrist et al (1997) used a visual search procedure to investigate grouping processes. They focused on the distinction between surface-based grouping (by manipulating the polarity of contrast of the elements that are to be grouped) and edge-based grouping (by manipulating the presence of collinear edges). The displays used by Gilchrist et al. had similar targets and distractors to those shown in Figure 1. Subjects in all cases were asked to detect the presence or absence of a vertical pair of items among a varying number of horizontal distractor pairs. The vertical target was present on half the trials and absent on the remaining half. By measuring reaction time (RT), and calculating the effect on reaction time of adding more distractors, an index of search efficiency results. Efficient search, where the target can be located relatively independently of the number of items in the display, suggests that the target is being detected in a spatially parallel manner- this has often been labelled feature search (e.g. Triesman, 1988). Search can also be dependent on the number of distractors in the display- conjunction or serial search. However, alternative explanations of search slope effects have been proposed (e.g. Duncan \& Humphreys, 1989; Humphreys \& Müller, 1993; Townsend, 1972).

Gilchrist et al. (1997), showed that, if the items had either common surface polarity of contrast or had collinear edges, then search was efficient and relatively independent of display size; however, if the items had opposite polarity 
(a)

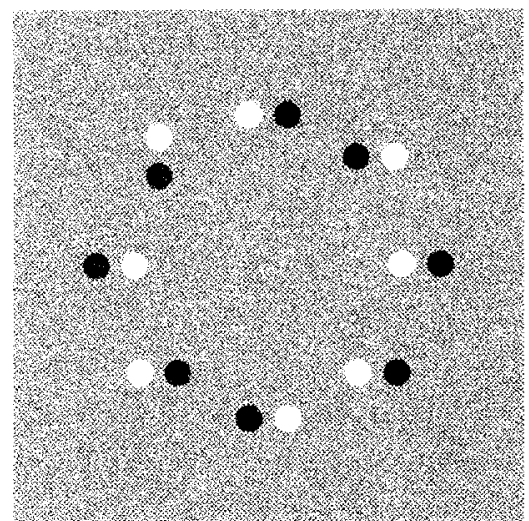

(c)

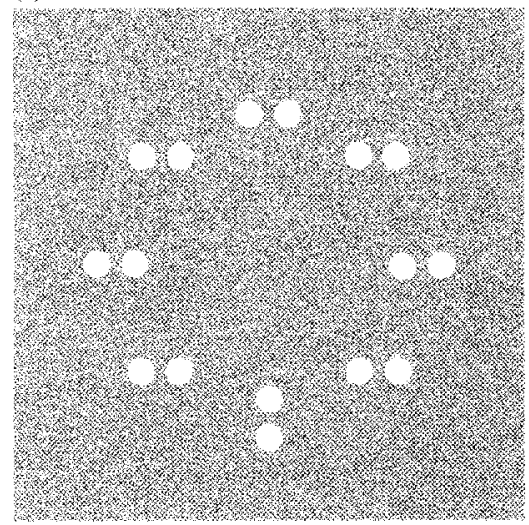

(b)

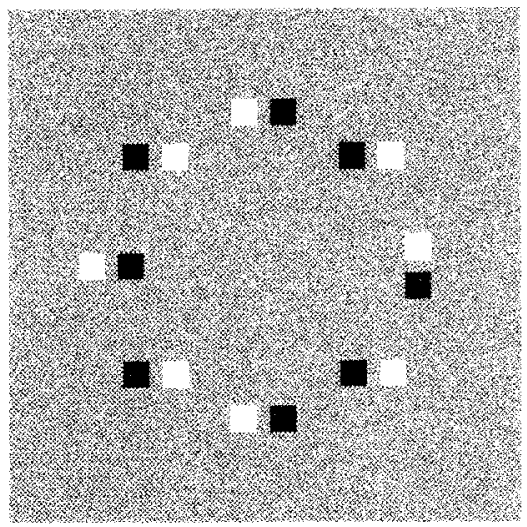

(d)

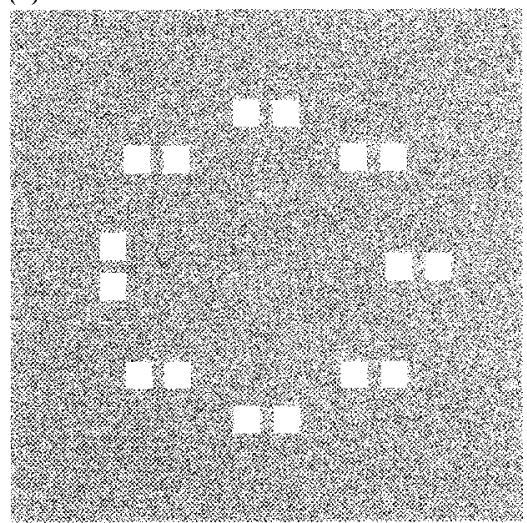

FIG. 1. Example displays for the four conditions in Experiment 1. The four conditions were Opposite polarity circles (a); Opposite polarity squares (b); Same polarity circles (c) and Same polarity squares (d).

of contrast and did not have collinear edges then search was inefficient and dependent on display size. The authors concluded that grouping was supported by an edge-based process that was contrast polarity insensitive and a surface-based process that was polarity sensitive.

\section{Spatial Integration and Saccadic Eye Movements}

Our approach in the present study was to find whether grouping affected subjects' eye movement responses in a search task. Saccadic eye movements are the main way by which visual attention is redirected and, in general, form an automatic component of tasks such as visual search. The programming of a 
saccade takes only a few hundred milliseconds and so study of their generation provides an alternative window into early visual processing.

One complication is an established form of spatial integration in the saccadic control system. The "global effect" describes the finding that when two items are presented in spatial proximity, saccades land at an intermediate position (Findlay, 1982; Ottes, Van Gisbergen, \& Eggermont, 1984). The integration involved in the global effect occurs with items within a spatial "window" of considerable extent (several degrees of visual angle). Ottes, Van Gisbergen, and Eggermont (1985) showed that, when subjects were instructed to search for targets defined by a specific colour, their initial saccade showed an influence of a neighbouring distractor of a different colour unless the saccade latency was considerably prolonged.

In our experiments, an arrangement was used (cf. Findlay, 1997) whereby global effect integration between target items and distractor items would be minimized. However, the target items were composed of pairs of elements in close proximity, for which strong global effect integration would be expected. Our interest was in whether, in spite of this, the grouping effects described previously would show some influence. Global effect grouping is not simply dependent on a low spatial frequency signal that "blurs" the items together. Thus, integration occurs between items of opposite contrast polarity (Findlay, Brogan, \& Wenban-Smith, 1993) and for isoluminant stimuli (Van Asten, Gielen, \& Winkel, 1988). Although it has been suggested that the global effect reflects a high-level strategy (He \& Kowler, 1989), its dependence on physical properties of the target (Deubel \& Hauske, 1988; Findlay et al., 1993) and its occurrence in more complex tasks (Findlay, 1997; Guez, Marchal, Le Gargasson, Grall, \& O'Regan, 1994) suggests that a combination of both sensory control and higher level strategies determine saccade landing position. Our experiment investigated whether information from edge-based and surface-based grouping would affect saccade programming.

Surprisingly little study has been made of eye movement control during visual search. In addition to the study by Ottes et al (1985) discussed earlier, Viviani and S wensson (1982) found that subjects were frequently unable to direct a saccade to a target when it was presented in the presence of neighbouring distractors (see also Zelinsky, 1996). Recent study by Findlay $(1995,1997)$ has shown that, provided target and distractors are separated by a larger region than the global effect window, a high proportion of accurate first saccades occurred (80-99\% for simple feature shape and colour search; $60-70 \%$ to a colour-shape conjunction).

\section{Current Study}

Here we studied the programming of saccades to targets in displays containing silmilar elements to those used by Gilchrist et al. (1997). We investigated 
whether the saccadic system can exploit representations in which items are encoded as larger structures and tested whether such processes are using edgeor surface-based processes for spatial integration. This contrasts with Gilchrist et al. where a RT target detection method was used. It is useful to note the two main differences in the demands places on the visual system by these two tasks. First, in the saccade selection task, subjects are required not only to detect the presence of the target but to localize the target spatially. Second, in the RT task, the subject is required to decide whether the target is present or not, whereas in the saccade selection task the target is al ways present. By comparing the relative contribution of edge- and surface-based factors in spatial integration across these two tasks a richer and more complete picture of how the visual system exploits these types of information can be built up.

In Experiment 1, we investigated the ability of subjects to make first saccades to pairs of items that grouped on the basis of common surface properties (same polarity of contrast circles) or collinear edges (opposite polarity of contrast squares). This was compared with a further two conditions in which neither grouping feature was present (opposite polarity circles) or both features were present (same polarity squares). Experiments 2 and 3 provide controls for two possible alternative explanations for the results of Experiment 1: First, that the effects are due to display heterogeneity (Experiment 2) or, second, due to the detection of an internal contour between the pairs of items (Experiment 3 ).

\section{GENERAL METHOD}

\section{Eye Movement Recording and Analysis}

Two-dimensional recordings of the right eye were made using a Fourward Technologies Dual Purkinje Image eyetracker (Crane \& Steele, 1985). The displays were viewed binocularly and head movements were minimized using a chin rest and two forehead rests. The analogue output from the eye tracker was sampled at $200 \mathrm{~Hz}$, and the eye position data was recorded on-line by the display computer.

The eye-movement data were analysed off-line by a semi-automatic procedure that detected the firstincidence of two successive samples registering a velocity over $25 \%$ sec. On occasional trials (less than $5 \%$ ), this algorithm detected small movements at the fixation that preceded the first saccade; in such cases the saccade onset position was selected manually. In addition, saccades with latencies of less than $100 \mathrm{msec}$ were excluded as were saccades that occurred after the duration of the trial $(1000 \mathrm{msec})$. The saccade landing position was taken as the eye position $80 \mathrm{msec}$ after the onset of the saccade. This avoids artefacts that result from lens displacement (Deubel \& Bridgeman, 1995). This procedure leads to two measures for each saccade: The time from display onset to the initiation of the saccade or saccade latency, and the landing position. The landing 
position was then recoded for saccade direction. In the current study the eight items (one target, seven distractors) were spaced uniformly around a circle, so saccades were classified as being in one of eight segments; a correct saccade was one that landed in the segment containing the target (target direction $\pm 22.5^{\circ}$ ).

\section{Procedure}

All blocks were preceded, and normally also succeeded, by a calibration procedure. This consisted of the subject being presented with a $3 \times 3$ grid of small crosses $\left(1 \times 1^{\circ}\right)$ which the subject was instructed to fixate in turn. The crosses were separated by $6^{\circ}$ in both the horizontal and vertical direction.

A single trial commenced with a central fixation box $\left(0.5 \times 0.5^{\circ}\right)$. After 1 second the fixation point was replaced by a display, which was displayed for a further 1 second. Eye position was sampled for the display duration as outlined earlier.

\section{Stimuli}

The stimuli were presented on a Macintosh 21-inch monitor, using purpose-written software and viewed at $57 \mathrm{~cm}$. For all three experiments the background of the display was $5.1 \mathrm{~cd} / \mathrm{m}^{2}$, all positive contrast items were $30.5 \mathrm{~cd} / \mathrm{m}^{2}$, and negative contrast items were $0.2 \mathrm{~cd} / \mathrm{m}^{2}$ as measured using a spot photometer.

In all three experiments the stimuli consisted of eight pairs of items evenly spaced in a circular display at $6^{\circ}$ eccentricity. Seven of the pairs of items were horizontally arranged and the eighth pair was vertically arranged (and constituted the target). In order to successfully make a saccade to the target, subjects would have to group the pairs of items together as both the target and the distractors were made up of the same two local elements (except in Experiment 3 where the items differed slightly).

\section{EXPERIMENT 1}

\section{Method}

Subjects. Five research workers acted as subjects. All had normal or corrected to normal vision. Three subjects had participated in eye-movements experiments before.

Procedure. Following a practice block of 16 trials, each subject participated in two blocks of 96 trials. The display conditions were presented randomly within each block. 
Stimuli. There were four display conditions. Two factors were varied: Element surface brightness and element shape (circles or squares). Elements could either have the same surface brightness (both lighter than the background) or different, opposite polarity, surface brightnesses (one lighter and one darker than the background). Opposite polarity squares had collinear edges to support grouping. Same polarity circles have the same surface property and same polarity squares have both collinear edges and the same surface property. Opposite polarity circles had neither the same surface property nor collinear edges to support grouping. These four conditions allow assessment of the contribution of edge and surface properties to the programming of saccades to groups of elements. Example displays from the four conditions in Experiment 1 are shown in Figure 1.

\section{Results}

The main variable considered in the current study was the ability to make a first saccade to the target. Two factors are important here: First, the number of saccades that were on target and, second, the latencies of these first saccades.

Two example saccade traces are shown in Figures 2 and 3. Figure 2 shows a first saccade made correctly to the target and Figure 3 shows a trial in which the first saccade was made to a distractor.

Table 1 shows the number of first saccades to target, for each subject, in each condition and the means across subjects are presented graphically in Figure 4. Overall accuracy was good $\left(51 \%\right.$ on target $\left.{ }^{1}\right)$, but there was wide variability across subjects (range from $37 \%$ to $74 \%$ on target), which may reflect a trade off between speed and accuracy (see below for a consideration of the saccade latencies).

TABLE 1:

The Number of First Saccades Directed Towards the Target (Target Location \pm 22.59 for Experiment 1.

\begin{tabular}{|c|c|c|c|c|c|}
\hline \multirow{3}{*}{$\frac{\text { Subject }}{\mathrm{SH}}$} & \multicolumn{2}{|c|}{$\begin{array}{c}\text { Opposite Polarity } \\
--------------\end{array}$} & \multicolumn{2}{|c|}{$\begin{array}{c}\text { Same Polarity } \\
-------------\end{array}$} & \multirow[b]{2}{*}{ Total } \\
\hline & Circles & Squares & Circles & Squares & \\
\hline & $14 / 47 \quad(30 \%)$ & $22 / 48 \quad(46 \%)$ & $30 / 48 \quad(63 \%)$ & $31 / 48 \quad(65 \%)$ & $97 / 191 \quad(51 \%)$ \\
\hline $\mathrm{KF}$ & $19 / 47 \quad(40 \%)$ & $22 / 45 \quad(49 \%)$ & $19 / 44 \quad(43 \%)$ & $27 / 46 \quad(59 \%)$ & $87 / 182 \quad(48 \%)$ \\
\hline $\mathrm{JF}$ & $14 / 48 \quad(29 \%)$ & $21 / 48 \quad(44 \%)$ & $30 / 48 \quad(63 \%)$ & $26 / 48 \quad(54 \%)$ & $91 / 192 \quad(47 \%)$ \\
\hline $\mathrm{FN}$ & $21 / 47 \quad(45 \%)$ & $35 / 48 \quad(73 \%)$ & $39 / 48 \quad(81 \%)$ & $46 / 48 \quad(96 \%)$ & $141 / 191 \quad(74 \%)$ \\
\hline SL & $14 / 48 \quad(29 \%)$ & $12 / 47 \quad(26 \%)$ & $19 / 48 \quad(40 \%)$ & $25 / 48 \quad(52 \%)$ & $70 / 191 \quad(37 \%)$ \\
\hline Total & $82 / 237(35 \%)$ & $112 / 236(47 \%)$ & $137 / 236(58 \%)$ & $155 / 238(65 \%)$ & $486 / 947 \quad(51 \%)$ \\
\hline
\end{tabular}

The percentage correct first saccades are shown in parentheses.

\footnotetext{
${ }^{1}$ Note that chance performance here would be $12.5 \%$ of first saccades on target.
} 

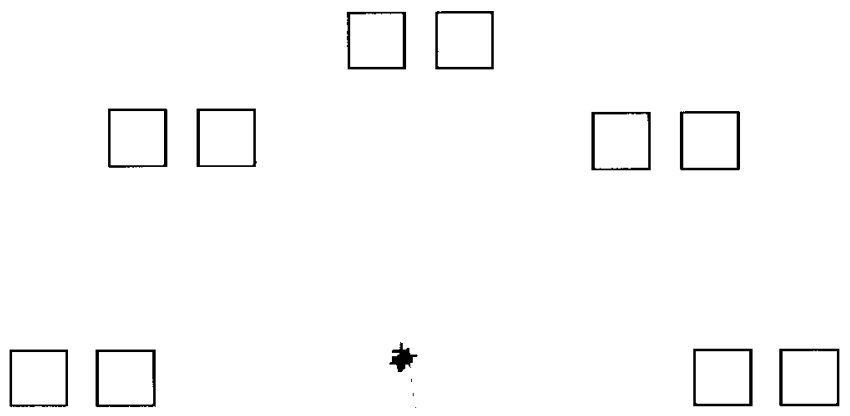

$+$
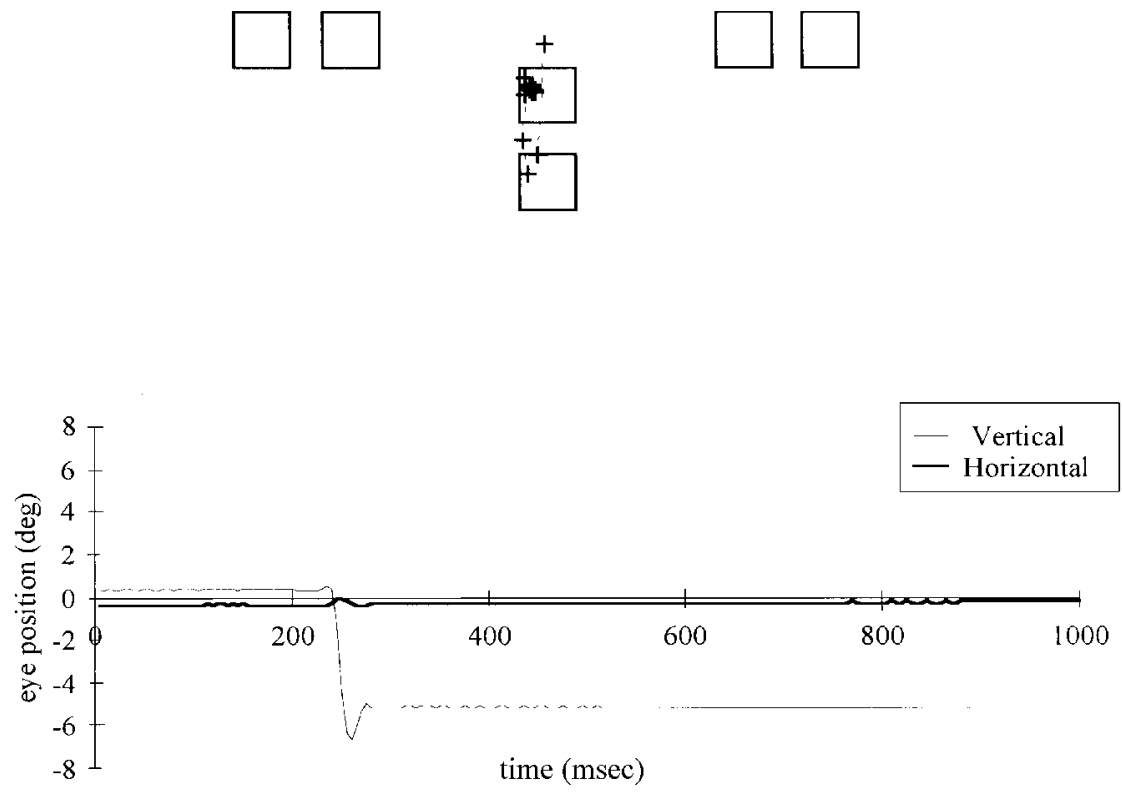

FIG. 2. Example saccade trace for a saccade that was correctly directed to the vertical target: Experiment 1 , subject SL, block 1 , trial 20 , same polarity squares.

The Opposite polarity circles condition provides the baseline performance when none of the grouping features was present. The five subjects show the worst performance (35\%) for this condition. With the addition of the collinear edge information (Opposite polarity squares), performance improved to $47 \%$. Performance was also improved (to $58 \%$ ) with the addition of same polarity surface information (Same polarity circles). The maximum increase in perfor- 
$\bigcirc$<smiles>C1CCCCCCC1</smiles><smiles>C1CCCCCC1</smiles>

$\bigcirc 0$
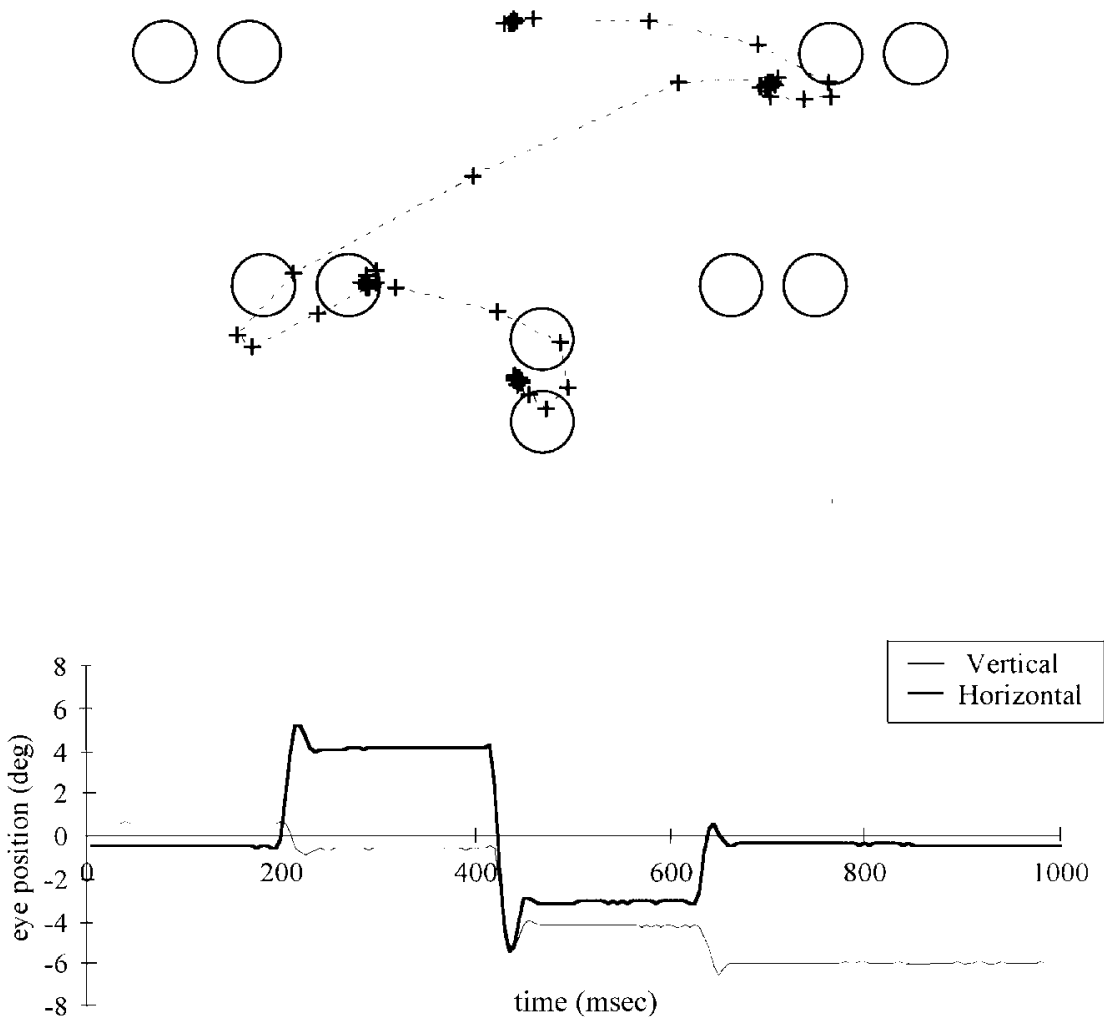

FIG. 3. Example saccade trace for a saccade that was incorrectly directed to the vertical target: Experiment 1, subject SL, block 1, trial 14, opposite polarity circles.

mance was found with the condition having both collinear edges and common surface features: $65 \%$ of first saccades were on target.

Inspection of the individual subject's performance shows that all subjects generally show the same pattern of performance. In four of the five subjects, performance for opposite polarity circles was worse than any of the other three conditions. Subject SL showed slightly worse performance for Opposite polarity squares than for Opposite polarity circles; however, this difference is not 


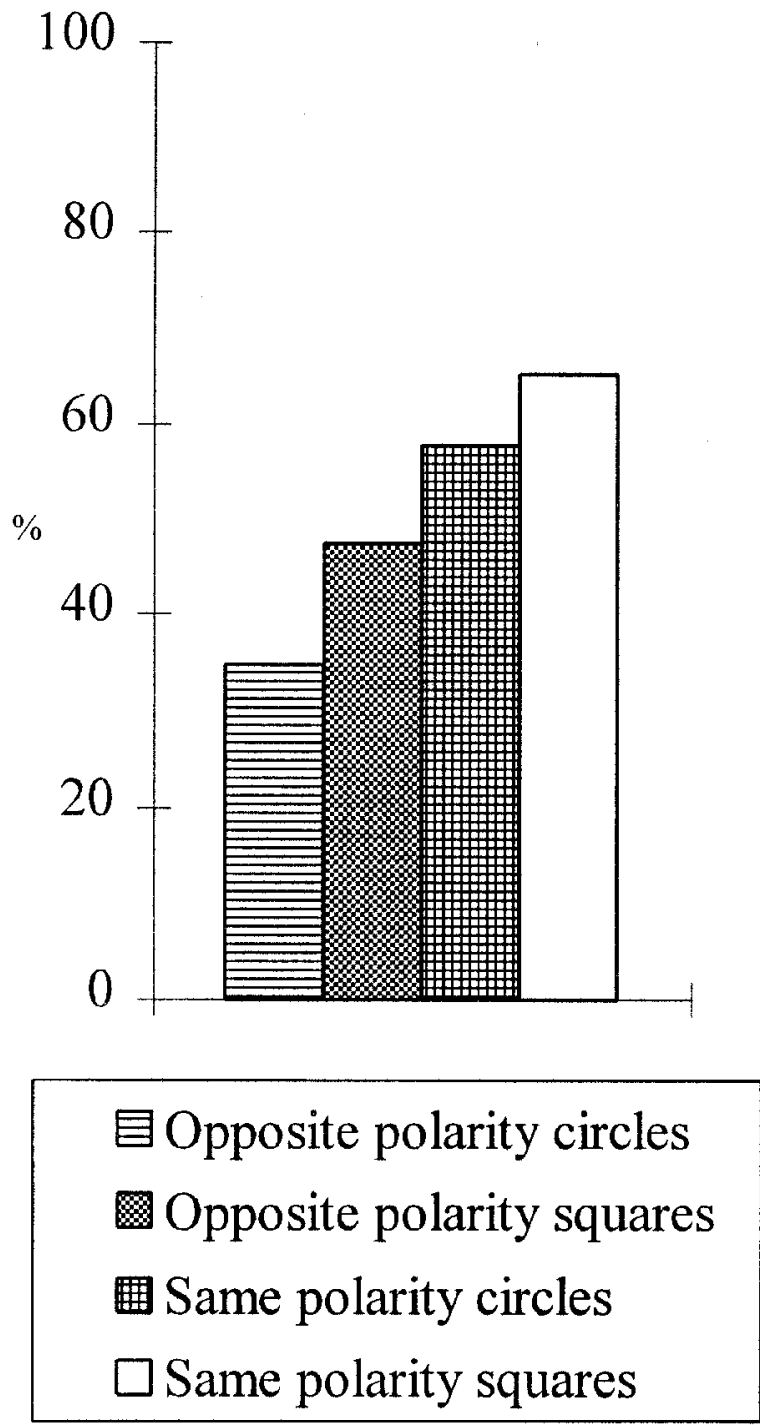

FIG. 4. The percentage of first saccades to target for the four conditions in Experiment 1.

significant. In addition, performance on the other two conditions was better than for the Opposite polarity circles.

Statistical analysis of the data was carried out using a log-linear analysis with Collinear edge (present vs. absent); Contrast polarity (same vs. different); Subject (SH, KF, JF, FN, SL); and Response (On target vs. Off target) as factors. Tests of partial association revealed a significant interaction for Contrast 
polarity $\times$ Response $^{2}: \chi^{2}(1)=43.4 ; P<.001$ and for Collinear edges $\times$ Response: $\chi^{2}(1)=10.3 ; P<.01$. However, there was no significant Contrast polarity $\times$ Collinear edge $\times$ Response interaction: $\chi^{2}(1)=.121$; ns. There were significant effects on performance of adding both collinear edges to the elements and changing the surface contrast polarity. The absence of an interaction between these two factors on performance shows that they combine additively to improve performance.

In addition there was a significant Response by Subject interaction: $\chi^{2}$ $(4)=62.3 ; P<.001$, reflecting the differences in overall performance levels among subjects. A significant Contrast Polarity $\times$ Subject $\times$ Response interaction was also found: $\chi^{2}(4)=10.4 ; P<.05$, which reflects differences among the subjects ability to utilize the surface information to aid grouping. The mean percentage difference between same and different polarity conditions was: $\mathrm{SH}$, $26 \%$; KF, 6.5\%; JF, 22\%; FN, 29.5\%; and SL, $18.5 \%$.

Saccade latency, the time from the onset of the display to the initiation of the saccade, provides a second measure of the load placed on the subject by the task. The saccade latencies for the four conditions across subjects are reproduced in Table 2.

Across subjects, the mean saccade latency varied widely (fastest subject overall SH: 230msec; slowest FN: 474msec). There does appear to be some relationship between saccade latency and target accuracy, with the slowest subject producing the most accurate saccades, possibly reflecting a strategy to slow down to improve accuracy. However, note that this does not appear to affect the pattern of saccade accuracy across conditions.

TABLE 2

The First Saccade Latencies for the Four Conditions in Experiment 1 for the Five Subjects

\begin{tabular}{|c|c|c|c|c|c|c|c|c|c|}
\hline \multirow[b]{2}{*}{ Subject } & \multicolumn{4}{|c|}{$\begin{array}{c}\text { Opposite Polarity } \\
----------------\end{array}$} & \multicolumn{4}{|c|}{$\begin{array}{l}\text { Same Polarity } \\
-----------\end{array}$} & \multirow[b]{2}{*}{ Average } \\
\hline & Mean & $S D$ & Mean & $S D$ & Mean & $S D$ & Mean & $S D$ & \\
\hline SH & 233 & 29 & 230 & 23 & 226 & 23 & 231 & 33 & 230 \\
\hline $\mathrm{KF}$ & 405 & 96 & 418 & 116 & 427 & 117 & 418 & 89 & 417 \\
\hline $\mathrm{JF}$ & 283 & 38 & 272 & 33 & 271 & 49 & 269 & 40 & 274 \\
\hline $\mathrm{FN}$ & 520 & 182 & 519 & 166 & 420 & 121 & 437 & 108 & 474 \\
\hline SL & 299 & 103 & 265 & 96 & 265 & 72 & 257 & 79 & 272 \\
\hline Average & 348 & & 341 & & 322 & & 322 & & - \\
\hline
\end{tabular}

${ }^{2}$ This is equivalent to what would be called, in a parametric analysis such as ANOVA, "a main effect" of Contrast polarity. 
There was no evidence that saccades were systematically slower across subjects for any condition. Saccades were slower overall for Opposite polarity items (squares $341 \mathrm{msec}$, circles $348 \mathrm{msec}$ ) than for Same polarity items (squares $322 \mathrm{msec}$, circles $322 \mathrm{msec}$ ); however, this is chiefly as a result of the large slowing for subject FN. These conclusions were confirmed using a within-subject repeated measure ANOVA, with Collinear edge (present vs. absent) and Contrast polarity (same vs. different) as factors. There was no significant effect of Collinear edge, $F(1,4)=1.55$, ns; Contrast polarity, $F(1$, $4)=0.44$, ns; or a Collinear edge $\times$ Contrast polarity interaction, $F(1$, 4) $=0.92$, ns.

\section{Discussion}

The accuracy of saccades to pairs of items that had to be grouped to form the target was dependent on two factors: First, the presence of collinear edge information and, second, on the presence of the same surface property across the items. This implies that the saccadic system has access to representations in which groups of items are formed into larger structures both on the basis of edge and surface information. In addition, at least for items of the same size and eccentricity used here, there is evidence that the surface characteristic is more important than the edge information, as surface information produces a greater reduction in the number of misdirected saccades (35\% to $58 \%$ ) than the addition of collinear edges ( $35 \%$ to $47 \%$ ).

These two factors, collinear edges and surface characteristic, combine additively. This is in contrast to the results reported by Gilchrist et al. (1997), where it was shown that these factors combined sub-additively. In the case of the RT data, adding both grouping factors produced no greater advantage in search performance than adding either one individually. These differences may reflect either: (1) A difference in the exact display characteristic; (2) a fundamental difference in the kind of processes that are involved in the computation of exact target location (as in saccadic programming) and the detection of the presence of the item, where no localization information is required by the task (as in an RT measure); or (3) simply reflect the lack of statistical power in current experiment to expose a Collinear edge $\times$ Contrast polarity interaction.

The current results can be summarized as follows: (1) Common surface properties aid grouping; and (2) collinearity aids grouping. However both effects could be explained by other features of the displays that did not depend on the grouping manipulation but instead on some additional cue that the shape or surface manipulation controls. These two explanations were investigated in Experiments 2 and 3.

For the manipulation of the surface feature, the opposite polarity vertical pair may be more difficult to detect because the displays are in general more heterogeneous. First, heterogeneity is increased because the target can be either 
white above black or black above white, but also because the distractors can be composed of black item on the left or on the right. Distractor heterog eneity is a factor that has been shown to influence search efficiency for both reaction time studies (Duncan, 1989; Duncan \& Humphreys, 1989) and saccade selection tasks (Findlay, 1995). In order to control for this factor, in Experiment 2 each display contained the same total number of black and white circles, as well as the same number of distractor types. In the Opposite polarity circles condition, items were paired one white with one black, so retaining opposite polarity within a pair (the displays were in fact identical to Experiment 1 ). In the second condition, circles were arranged in same polarity pairs; this resulted in half the pairs of items being black and half the pairs of items being white. The target was randomly white or black.

\section{EXPERIMENT 2}

Method

Subjects. Five research workers acted as subjects. All had corrected to normal vision and two had participated in Experiment 1.

Procedure. Subjects performed one block of 96 trials. The two display conditions were presented randomly within the block.

Stimuli. Example displays are shown in Figure 5. The opposite polarity condition was identical to Experiment 1 . The mixed same polarity condition

(a)

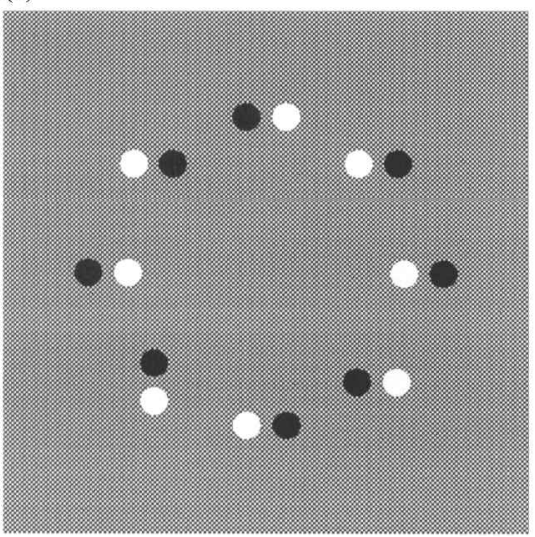

(b)

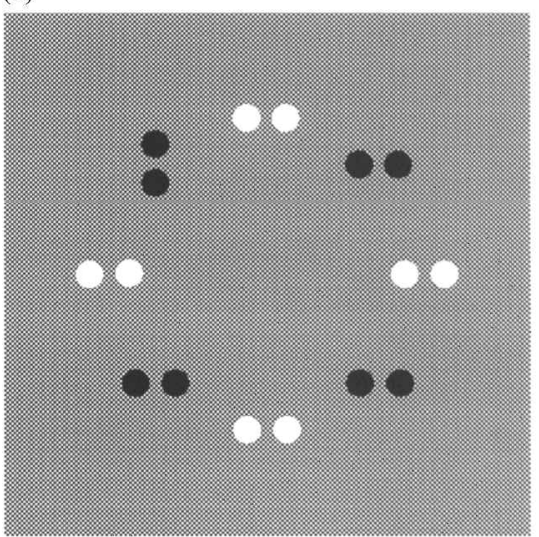

FIG. 5. Example displays for the two conditions in Experiment 2: (a) Opposite polarity circles and (b) mixed same polarity circles. 
contained an equal number of light and dark pairs, with the target being either dark or light, randomly, from trial to trial.

\section{Results}

The results are clear. All five subjects showed better accuracy in the directing of first saccades for the Mixed same polarity circles condition than for Opposite polarity circles condition. Overall the increase in performance was from $39 \%$ to $62 \%$. Figure 6 illustrates the mean across subjects graphically, and the individual results appear in Table 3.

Statistical analysis of the data was carried out using a log-linear analysis with Condition (Mixed same polarity circles vs. opposite polarity circles); Subject (SH, AS, EG, FN, BK) and Response (On target vs. Off target) as factors. Tests of partial association revealed significant interactions for Condition $\times$ Response: $\chi^{2} \quad(1)=27.4 ; \quad P<.001$ and Subject $\times$ Response: $\chi^{2}$ (4) $=61.3 ; P<.001$, which reflects differences in overall performance among subjects.

Saccade latencies are shown in Table 4. There was no systematic trend across subjects: Two subjects had longer latencies in the Opposite polarity condition, two shorter, and one the same. However, overall latencies were longer for opposite polarity circles $(315 \mathrm{msec})$ than for Mixed same polarity circles $(308 \mathrm{msec})$. These conclusions were confirmed using a within-subject repeated measure ANOVA, with Condition (Mixed same polarity circles vs. opposite polarity circles) as a factor. There was no significant effect of Condition, $F(1$, 4) $=0.46$, ns.

\section{Discussion}

Subjects showed a drop in performance for saccading to pairs of items that had opposite polarity even when compared with displays in which the total number of black and white items was kept the same. This suggests that difficulties

TABLE 3

The Number of First Saccades Directed Toward the Target (Target Location $\pm 2.5^{\circ}$ ) for Experiment 2 .

\begin{tabular}{|c|c|c|c|}
\hline Subject & $\begin{array}{c}\text { Mixed Same } \\
\text { Polarity Circles }\end{array}$ & $\begin{array}{c}\text { Opposite } \\
\text { Polarity Circles }\end{array}$ & Total \\
\hline $\mathrm{SH}$ & $35 / 48 \quad(73 \%)$ & $15 / 48 \quad(31 \%)$ & $25 / 96 \quad(26 \%)$ \\
\hline AS & $15 / 48 \quad(31 \%)$ & $10 / 48 \quad(21 \%)$ & $51 / 96 \quad(53 \%)$ \\
\hline EG & $25 / 48 \quad(52 \%)$ & $16 / 48 \quad(33 \%)$ & $41 / 96 \quad(43 \%)$ \\
\hline FN & $45 / 48 \quad(94 \%)$ & $29 / 46 \quad(63 \%)$ & $74 / 94 \quad(79 \%)$ \\
\hline BK & $28 / 48 \quad(58 \%)$ & $23 / 48 \quad(48 \%)$ & $50 / 96 \quad(52 \%)$ \\
\hline Total & $148 / 240(62 \%)$ & $93 / 238(39 \%)$ & $241 / 478(50 \%)$ \\
\hline
\end{tabular}

The percentage correct first-saccades are shown in parentheses. 


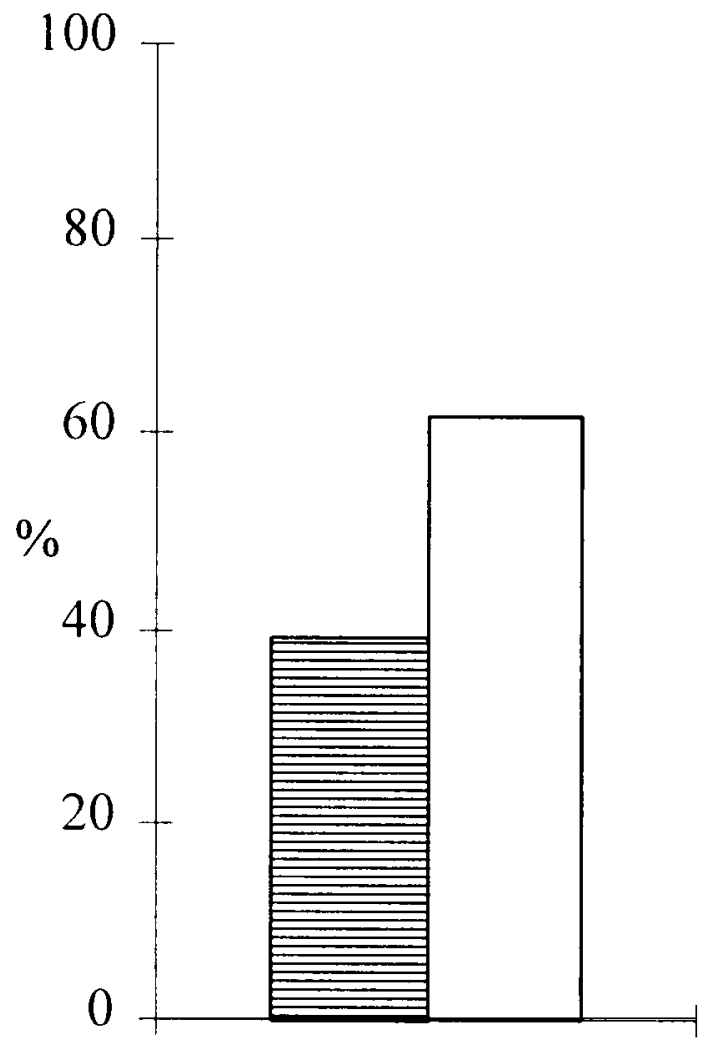

\section{目 Opposite polarity circles $\square$ Mixed same polarity circles}

FIG 6. Percentage of first saccades to target for the two conditions in Experiment 2.

encountered when grouping items that have different surface properties is not a result of overall display heterogeneity (cf. Duncan \& Humphreys, 1989). The current experiment, in conjunction with the results of Experiment 1, suggests that surface information is important in the grouping of items.

In Experiment 3 we investigated an alternative explanation for the advantage found when substituting circles for squares. One possible explanation for the improved performance with the addition of collinear edge information is that, by changing the shape from circles to squares, a strong internal horizontal 
TABLE 4

The First Saccade Latencies for the Two Conditions in

Experiment 2 for the Five Subjects

\begin{tabular}{|c|c|c|c|c|c|}
\hline \multirow[b]{2}{*}{ Subject } & \multicolumn{2}{|c|}{$\begin{array}{c}\text { Mixed Same } \\
\text { Polarity Circles } \\
-------\end{array}$} & \multicolumn{2}{|c|}{$\begin{array}{c}\text { Opposite } \\
\text { Polarity Circles } \\
-------\end{array}$} & \multirow[b]{2}{*}{ Average } \\
\hline & Mean & $S D$ & Mean & $S D$ & \\
\hline $\mathrm{SH}$ & 240 & 32.0 & 240 & 30.2 & 240 \\
\hline AS & 243 & 55.1 & 226 & 55.3 & 235 \\
\hline EG & 276 & 42.2 & 275 & 43.4 & 276 \\
\hline $\mathrm{FN}$ & 455 & 114.5 & 515 & 167.3 & 485 \\
\hline BK & 323 & 55.5 & 326 & 68.9 & 325 \\
\hline Average & 308 & & 315 & & 311 \\
\hline
\end{tabular}

contour is introduced between the pairs of items. Experiment 3 is a test of whether the improved performance with the addition of collinear edges is in fact a result of subjects detecting the internal horizontal contour in the target, which for the distractor items is vertical. In Experiment 3 we compare performance between Opposite polarity circles (as in Experiment 1) and pairs of wedge shapes in which the internal contour is always oriented at $45^{\circ}$.

\section{EXPERIMENT 3}

\section{Method}

Subjects. The five subjects who participated in Experiment 2 also participated in this experiment. The order of testing between Experiments 2 and 3 was counterbalanced as far as possible.

Procedure. The procedure was identical to Experiment 2, with each subject participating in one block of 96 trials and with the two conditions randomised within the single block.

Stimuli. There were two conditions: Opposite polarity circles (as in Experiment 1) and Opposite polarity wedges. The opposite polarity wedges, whether target or distractor, were constructed such that the internal contour was al ways at $45^{\circ}$ orientation (from north-east to south-west). Example displays from the two conditions are shown in Figure 7. The ordering of the polarity of the individual items was randomized, and identical in the two conditions.

\section{Results}

Again, the results are clear: All five subjects made more first correct saccades in the Opposite polarity wedge condition than in the Opposite polarity circle 
(a)

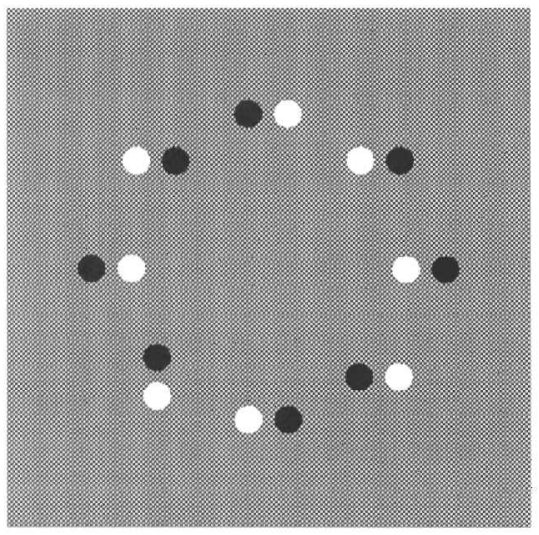

(b)

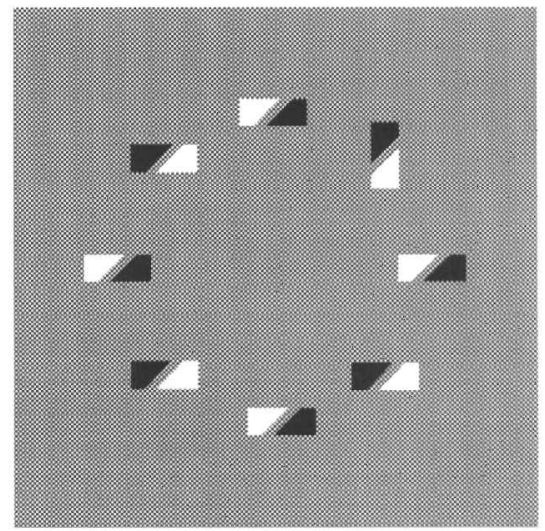

FIG. 7. Example displays for the two conditions in Experiment 3: (a) Opposite polarity circles and (b) opposite polarity wedges.

condition, with performance improving from $41 \%$ to $61 \%$ of first saccades on target. As before, the overall levels of performance among subjects was quite varied (range from $31 \%$ to $80 \%$ first saccades on target); however, the difference in performance, between conditions, remained unaffected. These overall results are illustrated in Figure 8 and the individual results appear in Table 5. Statistical analysis of the data was carried out using a log-linear analysis with Condition (Opposite polarity wedges vs. Opposite polarity circles); Subject (SH, AS, EG, FN, BK); and Response (On target vs. Off target) as factors. Tests of partial association revealed significant interactions for Condition $\times \mathrm{Re}$ sponse: $\chi^{2}(1)=23.6 ; \quad P<.001$ and Subject $\times$ Response: $\chi^{2}(4)=56.6$; $P<.001$, which reflects differences in overall performance between subjects.

The saccade latencies for each subject appear in Table 6. Saccades were slower overall for the Opposite polarity circles, with the mean difference

\section{TABLE 5}

The Number of First Saccades Directed Toward the Target (Target Location \pm 22.59 for Experiment 3 .

\begin{tabular}{|c|c|c|c|}
\hline Subject & $\begin{array}{c}\text { Opposite } \\
\text { Polarity Circles }\end{array}$ & $\begin{array}{c}\text { Opposite } \\
\text { Polarity Wedges }\end{array}$ & Total \\
\hline SH & $27 / 48 \quad(56 \%)$ & $24 / 48 \quad(50 \%)$ & $66 / 132(50 \%)$ \\
\hline AS & $45 / 65 \quad(69 \%)$ & $21 / 67 \quad(31 \%)$ & $40 / 96 \quad(42 \%)$ \\
\hline EG & $21 / 48 \quad(44 \%)$ & $8 / 47 \quad(17 \%)$ & $29 / 95 \quad(31 \%)$ \\
\hline $\mathrm{FN}$ & $42 / 48 \quad(88 \%)$ & $34 / 47 \quad(72 \%)$ & $76 / 95 \quad(80 \%)$ \\
\hline BK & $22 / 48 \quad(46 \%)$ & $18 / 48 \quad(38 \%)$ & $51 / 96 \quad(53 \%)$ \\
\hline Total & $105 / 257(41 \%)$ & $157 / 257(61 \%)$ & $262 / 514(51 \%)$ \\
\hline
\end{tabular}

The percentage correct first saccades are shown in parenthesis. 


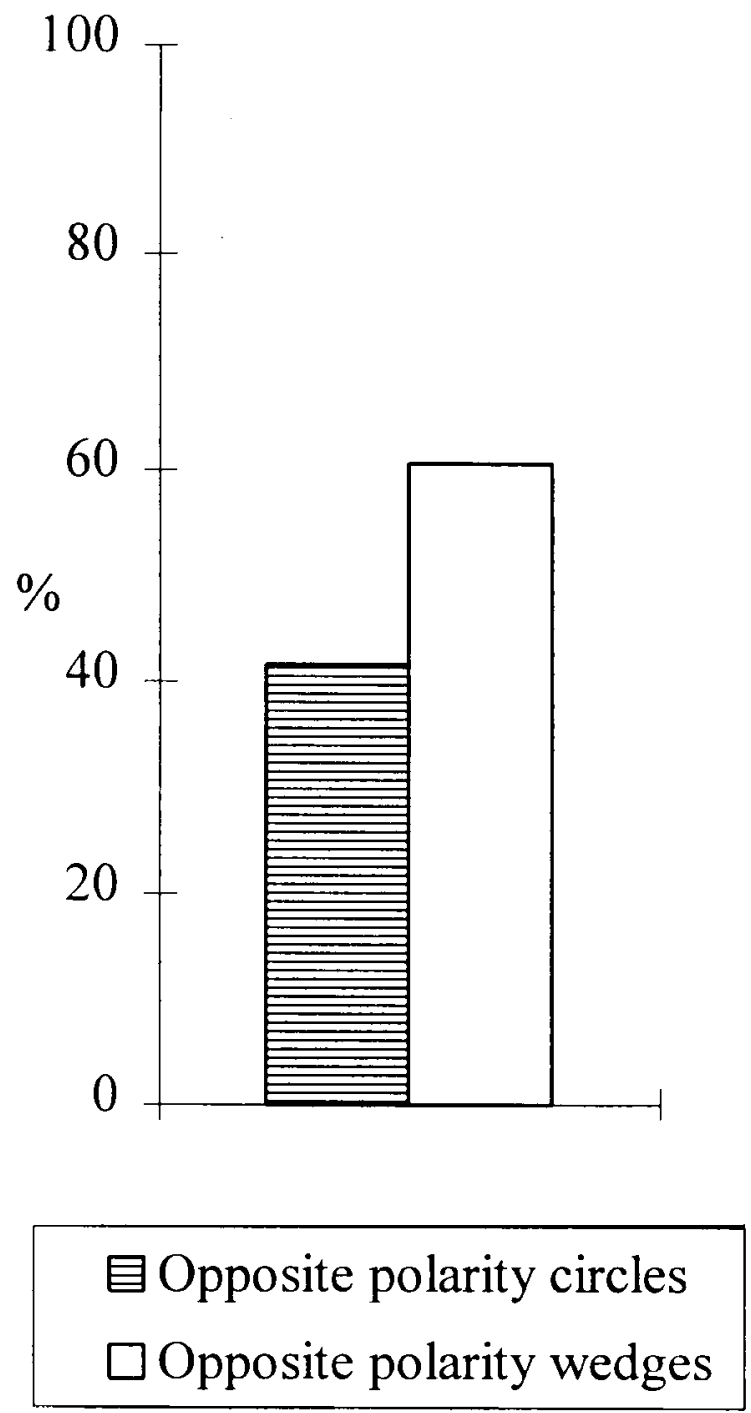

FIG. 8. The percentage of first saccades to target for the two conditions in Experiment 3.

between the two conditions being $26 \mathrm{msec}$. All five subjects showed positive, but varying amounts of slowing. This increase in saccade latency may have led to a decrease in the size of the difference observed in saccade accuracy. Statistical analysis was carried out using a within-subject repeated measure ANOVA, with Condition (Opposite polarity wedges vs. Opposite polarity circles) as a factor. There was no significant effect of Condition, $F(1,4)=4.73$, ns. Although, there was a strong trend $(P=.095)$. 
TABLE 6

The First Saccade Latencies for the Two Conditions in Experiment 3 for the Five Subjects

\begin{tabular}{llrlrl}
\hline & \multicolumn{2}{c}{$\begin{array}{c}\text { Opposite } \\
\text { Polarity Wedges }\end{array}$} & \multicolumn{2}{c}{$\begin{array}{c}\text { Opposite } \\
\text { Polarity Circles }\end{array}$} \\
& \multicolumn{2}{c}{ Subject } & Mean & SD & \multicolumn{2}{c}{ Mean } & SD & Average \\
\hline SH & 228 & 31.8 & 242 & 33.1 & 235 \\
AS & 252 & 39.2 & 261 & 52.8 & 257 \\
EG & 276 & 31.0 & 281 & 45.4 & 279 \\
FN & 475 & 150.0 & 546 & 167.3 & 511 \\
BK & 289 & 87.8 & 322 & 92.7 & 306 \\
Average & 304 & & 330 & & 317 \\
\hline
\end{tabular}

\section{Discussion}

The current experiment confirms that the effect, on saccade accuracy, of changing the shapes in Experiment 1 from circles to squares, was not a result of the introduction of a strong internal contour between the items that gave a unique cue to target location. Here this contour was removed and the addition of collinear edge information still lead to a significant improvement in search performance. Even though subjects were slower to make their first saccades in the opposite polarity condition, this did not prove to be sufficient to improve accuracy. Collinear edge information appears to aid in the integration of items regardless of whether the items have the same polarity or opposite polarity.

\section{GENERAL DISCUSSION}

Our experiments used saccadic response as an indicator of early visual processing, using a variety of types of display containing two element target and distractor pairs. The target was defined in all cases only by the interrelation between the parts. In comparison with the baseline condition of opposite polarity circles, the ability to saccade to the vertically oriented target pair from a set of horizontally oriented distractor pairs was enhanced by the inclusion of either collinear edge or common surface properties. Experiments 2 and 3 were control experiments to test alternative explanations for the results. Experiment 2 tested whether the effect of changing the surface properties was a result of the change in display heterogeneity, or a result of the polarity difference within each pair. The results showed that even when there was heterogeneity between items, and the number of white and black dots was kept constant, the number of saccades to target was higher when, within each pair, the items had the same or different surface properties. Experiment 3 tested whether the improved performance with the addition of collinear edge information in Experiment 1 was a result of 
the introduction of a strong internal contour between the items. The experiment showed that even when this contour does not provide a strong cue to target location saccade accuracy is still improved by the introduction of collinear edge information.

The current results suggest that spatial integration does occur between items for saccade production. The efficiency of this integration process is compromised when the two items do not share either common surface properties or collinear edges. Such processes may facilitate saccades to objects in everyday viewing- the presence of common surface properties, or collinear edges is a good cue that the two parts belong to a common structure or object.

In a similar search study, Findlay (1997) asked subjects to saccade to a red target amongst green distractors. On some trials two red targets were presented in neighbouring locations and subjects often made saccades to a mid point between the two items. This result demonstrated that under some circumstances, elements in a multi-element display are indeed processed in groups, even when the task does not explicitly require it. Indeed, such integration is an integral part of many visual search theories (e.g. Duncan \& Humphreys, 1989).

It is clear from these data that neither an edge-based theory of the visual information that controls saccadic programming, nor a surface-based theory, can alone be sufficient. In addition it is clear that in search conditions, when a target must be located in the presence of distractoritems, spatial integration processes can operate to treat a number of elements as a structural unit.

In the current experiments we can only claim that the surface manipulation is a result of using the same or different surface luminance. Gilchrist et al. (1997) made a systematic study of the parameters that control the surface similarity; and they found that grouping was disrupted specifically by the change in contrast polarity and not by the existence of a luminance difference alone. However, it is beyond the scope of the current study to discern whether for saccade programming it is contrast polarity per se that disrupts grouping.

Grossberg and colleagues (e.g. Grossberg, 1994; Grossberg \& Mingolla, 1985 ) have developed a systematic model to explain the grouping of items into larger structures. The model consists of a Feature Contour System (FCS) and a Boundary Contour System (BCS). The BCS extracts edge information and groups regions on the basis of this information. This system is contour polarity insensitive: A light-to-dark edge is processed in a similar way as a dark-to-light edge. Within this system, collinear edges are grouped and this grouping would occur independently of the polarity of the items; such a mechanism would allow the grouping of the opposite polarity squares in the current experiments. The second system, the FCS, processes surface information. Within this system, activity spreads on the basis of the surface information. This process is contrast polarity dependent and so would allow the grouping of common surface regions, but not regions that have opposite contrast polarity. The two systems are not in fact independent because the spread of the signals in the FCS is 
stopped by the BCS boundary signals. In tandem, these two system produce visual representations in which coherent bounded regions are formed. Such a system could explain the current results well. The BCS is responsible for the grouping based on collinear edge information, and, as the BCS is contrast polarity independent, grouping of this kind can occur for opposite polarity as well as same polarity items. Surface-based grouping occurs within the FCS and so is contrast polarity dependent: Opposite contrast polarity items do not group efficiently, but same brightness items do group efficiently.

Although the FCS/BCS architecture provides a compelling model for the current results a number of caveats are worth noting here. First, this architecture does not provide an explanation for how these early processes lead to the detection of a target item among distractors- the model only describes how the display could be segmented into horizontal elements amongst vertical items. Second, performance with the opposite polarity circles was not at chance in any of the three experiments; this implies that the subjects had partial information about the target location even in the case when the items shared neither collinear edges nor common surface properties. There are two possible explanations for this. The first is that subjects made covert shifts of attention around candidate target locations until they located the target and then made a saccade to the target. If this strategy was only partly successful, then this would explain subjects above chance, but poor, performance with opposite polarity circles. Such a process should also lead to an increase in latency for the opposite polarity condition, but this was not a consistent finding across subjects or experiments. A second possibility is that an inefficient binding of the opposite polarity circles is possible, but that this processes only leads to a successful correct localization of the target on occasional trials.

Whatever the exact processes that underlie the differences between the poor integration of opposite polarity circles and the improvement with the addition of collinear edges or common surface properties, such processes do appear to be involved in the spatial integration of items into larger structures both for item detection (Gilchrist et al., 1997) and for the locating of items by the saccadic system as reported here.

\section{REFERENCES}

Crane, J.D., \& Steele, C.M. (1985). Generation-V dual-Purkinje-image eyetracker. Applied Optics, 24, 527-537.

Deubel, H., \& Bridgeman, B. (1995). Fourth Purkinje image signals reveal eye-lens deviations and retinal image distortions during saccades. Vision Research, 35(4), 529-538.

Deubel, H., \& Hauske, G. (1988). The programming of visually guided saccades. In H. Marko, G. Hauske, and A. Struppler (Eds.), Processing structures for perception and action (pp. 67-74). Weinheim, Germany: Verlag Chemie.

Duncan, J. (1989). Boundary-conditions on parallel processing in human-vision. Perception, 18(4), 457-469. 
Duncan, J., \& Humphreys, G.W. (1989). Visual search and stimulus similarity. Psychological Review, 96, 433-458.

Findlay, J.M. (1982). Global processing for saccadic eye movements. Vision Research, 22, 1033-1045.

Findlay, J.M. (1995). Visual search: Eye movements and peripheral vision. Optometry and Vision Science, 72, 461-466.

Findlay, J.M. (1997). Saccade target selection during visual search. Vision Research, 37, $617-631$.

Findlay, J.M., Brogan, D., \& Wenban-Smith, M. (1993). The visual signal for saccadic eye movements emphasises visual boundaries. Perception and Psychophysics, 53, 633-641.

Gilchrist, I.D., Humphreys, G.W., Riddoch, M.J., \& Neumann, H. (1997). Luminance and edge information in grouping: A study using visual search. Journal of Experimental Psychology: Human Perception and Performance, 23, 464-480.

Grossberg, S. (1994). 3-D vision and figure ground separation by visual-cortex. Perception and Psychophysics. 55(1), 48-120.

Grossberg, S., \& Mingolla, E. (1985). Neural dynamics of perceptual grouping: Texture, boundaries, and emergent segmentation. Perception and Psychophysics, 38(2), 141-171.

Guez, J-E., Marchal, P., Le Gargasson, J-F., Grall, Y., \& O' Regan, J. K. (1994). Eye fixation near corners: Evidence for a centre of gravity calculation based on contrast, rather than luminance or curvature. Vision Research, 34(12), 1625-1635.

He, P., \& Kowler, E. (1989). The role of location probability in the programming of saccades: Implications for "center-of-gravity" tendencies. Vision Research, 29, 1165-1181

He, Z.J., \& Nakayama, K. (1992). Surface versus features in visual search. Nature, 359, 231-233.

Humphreys, G.W., \& Müller, H.J. (1993). SEarch via Recursive Rejection (SERR): A connectionist model of visual search. Cognitive Psychology, 25, 43-110.

Marr, D., \& Hildreth, E. (1980). Theory of edge detection. Proceedings of the Royal Society of London, B, 207, 269-294.

Ottes, F.P., van Gisbergen, J.A.M., \& Eggermont, J.J. (1984). Metrics of saccade responses to visual double stimuli: Two different modes. Vision Research, 24, 1167-1179.

Ottes, F.P., van Gisbergen, J.A.M., \& Eggermont, J.J. (1985). Latency dependence of colour-based target vs nontarget discrimination by the saccadic system. Vision Research, 25, $849-862$.

Townsend, J.T. (1972). Some results on the identification of parallel and serial processes. British Journal of Mathematical and Statistical Psychology, 25, 168-199.

Triesman, A. (1988). Features and objects: The Fourteenth Bartlett Memorial Lecture. The Quarterly Journal of Experimental Psychology, 40A(2), 201-237.

Van Asten, W.N.J.C., Gielen, C.C.A.M., \& Winkel, M.E.M. (1988). The effect of isoluminant and ischromatic stimuli on latency and amplitude of saccades. Vision Research, 28(7), $827-840$.

Viviani, P., \& Swensson, R.G. (1982). Saccadic eye movements to peripherally discriminated visual targets. Journal of Experimental Psychology: Human Perception and Performance, 8, 113-126.

Watt, R.J., \& Morgan, M.J. (1985). A theory of the primitive spatial code in human vision. Vision Research, 25(11), 1661-1674.

Zelinsky, G.J. (1996). Using eye saccades to assess the selectivity of search movements. Vision Research, 36(14), 2177-2187. 